\title{
Monoids, their boundaries, fractals and $C^{\star}$-algebras
}

https://doi.org/10.1515/taa-2020-0003

Received March 11, 2019; accepted January 31, 2020

Abstract: In this note we establish some connections between the theory of self-similar fractals in the sense of John E. Hutchinson (cf. [3]), and the theory of boundary quotients of $C^{\star}$-algebras associated to monoids. Although we must leave several important questions open, we show that the existence of self-similar $\mathscr{M}$ fractals for a given monoid $\mathscr{M}$, gives rise to examples of $C^{\star}$-algebras (1.9) generalizing the boundary quotients $\partial C_{\lambda}^{\star}(\mathscr{M})$ discussed by X. Li in [4, $\$ 7$, p. 71]. The starting point for our investigations is the observation that the universal boundary of a finitely 1-generated monoid carries naturally two topologies. The fine topology plays a prominent role in the construction of these boundary quotients, while the cone topology can be used to define canonical measures on the attractor of an $\mathscr{M}$-fractal for a finitely 1-generated monoid $\mathscr{M}$.

Keywords: Monoids, boundaries, fractals, $C^{\star}$-algebras

MSC: 20M30, 47D03, 28A80

\section{Introduction}

On a monoid $\mathscr{M}$ (=semigroup with unit $1_{\mathscr{M}}$ ) there is naturally defined a reflexive and transitive relation “ $\preceq$ ”, i.e., for $\omega, \tau \in \mathscr{M}$ one defines $\omega \preceq \tau$ if, and only if, there exists $\sigma \in \mathscr{M}$ satisfying $\omega=\tau \cdot \sigma$. In particular, one may consider $\left(\mathscr{M}, \preceq\right.$ ) as a partially ordered set. Moreover, if $\mathscr{M}$ is $\mathbb{N}_{0}$-graded, then $(\mathscr{M}, \preceq)$ is a (noetherian) partially ordered set (see Corollary 3.7). Such a poset has a poset completion $i_{\mathscr{M}}: \mathscr{M} \longrightarrow \overline{\mathscr{M}}$ (see § 2.3), and one defines the universal boundary $\partial \mathscr{M}$ of $\mathscr{M}$ by

$$
\partial \mathscr{M}=(\overline{\mathscr{M}} \backslash \operatorname{im}(i \mathscr{M})) / \approx,
$$

where $\approx$ is the equivalence relation induced by " $\preceq$ " on $\overline{\mathscr{M}} \backslash \operatorname{im}\left(i_{\mathscr{M}}\right)$ (see $\S 2.3$ ). For several reasons (cf. Theorem $\mathrm{A}$, Theorem B, Theorem C) one may consider $\partial \mathscr{M}$ as the natural boundary associated to the monoid $\mathscr{M}$. However, it is less clear what topology one should consider. Apart from the cone topology $\mathscr{T}_{c}(\overline{\mathscr{M}})$ there is another potentially finer topology $\mathscr{T}_{f}(\overline{\mathscr{M}})$ which will be called the fine topology on $\partial \mathscr{M}$ (cf. $\S$ 2.6), i.e., the identity

$$
\operatorname{id}_{\partial \mathscr{M}}:\left(\partial \mathscr{M}, \mathscr{T}_{f}(\overline{\mathscr{M}})\right) \longrightarrow\left(\partial \mathscr{M}, \mathscr{T}_{c}(\overline{\mathscr{M}})\right)
$$

is a continuous map. The monoid $\mathscr{M}$ will be said to be $\mathscr{T}$-regular, if (1.2) is a homeomorphism. E.g., finitely generated free monoids are $\mathscr{T}$-regular (cf. Proposition 3.11, § 4.1). The universal boundary $\widetilde{\mathscr{M}}=\left(\partial \mathscr{M}, \mathscr{T}_{f}(\mathscr{M})\right.$ with the fine topology can be identified with the Laca boundary $\widehat{E}(\mathscr{M})$ of the monoid $\mathscr{M}$. This topological space plays an essential role for defining boundary quotients of $C^{\star}$-algebras associated to monoids (cf. [4, $\S 7]$, [5]). Indeed one has the following (cf. Theorem 3.10).

Theorem A. The map $\bar{\chi} .:\left(\partial \mathscr{M}, \mathscr{T}_{f}(\overline{\mathscr{M}})\right) \longrightarrow \widehat{E}(\mathscr{M})$ defined by (3.21) is a homeomorphism.

\footnotetext{
*Corresponding Author: Giulia dal Verme: Dipartimento di Matematica e Applicazioni, Università degli Studi di MilanoBicocca, Ed. U5, Via R.Cozzi 55, 20125 Milano, Italy, E-mail: giulia.dalverme@unimib.it

Thomas Weigel: Dipartimento di Matematica e Applicazioni, Università degli Studi di Milano-Bicocca, Ed. U5, Via R.Cozzi 55, 20125 Milano, Italy, E-mail: thomas.weigel@unimib.it
} 
Remark 1.1. (a) By Theorem A, the topological space $\left(\partial \mathscr{M}, \mathscr{T}_{f}(\overline{\mathscr{M}})\right)$ is totally-disconnected and compact and thus it has the nicest topological regularity property that one can wish for. On the contrary, in general one can only show that $\left(\partial \mathscr{M}, \mathscr{T}_{c}(\overline{\mathscr{M}})\right)$ is a $T_{0}$-space, which is a low level regularity property. Indeed, if $\left(\partial \mathscr{M}, \mathscr{T}_{c}(\overline{\mathscr{M}})\right)$ happens to be Hausdorff, then (1.2) is necessarily a homeomorphism and $\mathscr{M}$ is $\mathscr{T}$-regular (cf. Proposition 3.11). We will now work with the cone topology $\mathscr{T}_{c}(\overline{\mathscr{M}})$.

(b) If $\phi: \mathscr{Q} \longrightarrow \mathscr{M}$ is a surjective graded homomorphism of finitely 1-generated monoids, then, by construction, $\phi$ induces a surjective, continuous and open map

$$
\partial \bar{\phi}:\left(\partial \mathscr{Q}, \mathscr{T}_{c}(\overline{\mathscr{Q}})\right) \longrightarrow\left(\partial \mathscr{M}, \mathscr{T}_{c}(\overline{\mathscr{M}})\right)
$$

(cf. Proposition 3.2). This property can be used to establish the following.

Theorem $B$. Let $\mathscr{M}$ be a finitely 1-generated $\mathbb{N}_{0}$-graded monoid. Then $\partial \mathscr{M}$ carries naturally a Borel probability meausure

$$
\mu_{\mathscr{M}}: \operatorname{Bor}(\partial \mathscr{M}) \longrightarrow \mathbb{R}^{+} \cup\{\infty\}
$$

induced by the canonical homomorphism of monoids $\phi_{\mathscr{M}}: \mathscr{F}\left\langle\mathscr{M}_{1}\right\rangle \longrightarrow \mathscr{M}(c f$. (3.4)).

On the other hand the induced mapping $\phi_{\widehat{E}}$ is given by a map

$$
\phi_{\widehat{E}}: \widehat{E}(\mathscr{M}), \longrightarrow \widehat{E}(\mathscr{Q}),
$$

(cf. Proposition 3.12). Hence for the purpose of constructing Borel measures the fine topology seems to be inappropriate.

Theorem B can be used to define the $C^{\star}$-algebra

$$
C^{\star}\left(\mathscr{M}, \mu_{\mathscr{M}}\right)=\left\langle\beta_{\omega}, \beta_{\omega}^{\star} \mid \omega \in \mathscr{M}\right\rangle \subseteq \mathcal{B}\left(L^{2}\left(\partial \mathscr{M}, \mathbb{C}, \mu_{\mathscr{M}}\right)\right)
$$

for every finitely 1 -generated $\mathbb{N}_{0}$-graded monoid $\mathscr{M}$, where $\beta(\omega)$ is the mapping induced by left multiplication with $\omega$ (cf. § 4.5). We will show by explicit calculation that for the monoid $\mathscr{F}_{n}$, freely generated by a set of cardinality $n$, the $C^{\star}$-algebra $C^{\star}\left(\mathscr{F}_{n}, \mu_{\mathscr{F}_{n}}\right)$ coincides with the Cuntz algebra $\mathcal{O}_{n}$ (cf. Proposition 4.4), while for the right-angled Artin monoid $\mathscr{M}^{\Gamma}$ associated to the finite graph $\Gamma, C^{\star}\left(\mathscr{M}^{\Gamma}, \mu_{\mathscr{M}^{\Gamma}}\right)$ coincides with the boundary quotients introduced by Crisp and Laca in [2] (cf. § 4.6). Nevertheless the following more general question remains unanswered.

Question 1. Let $\mathscr{M}$ be a finitely 1-generated $\mathbb{N}_{0}$-graded monoid with the left cancellation property. Does $C^{*}\left(\mathscr{M}, \mu_{\mathscr{M}}\right)$ coincide with the boundary quotient $\partial C_{\lambda}(\mathscr{M})$ defined by X. Li in [4, Definition 7.9]?

From now on we will assume that the $\mathbb{N}_{0}$-graded monoid $\mathscr{M}=\bigcup_{k \in \mathbb{N}_{0}} \mathscr{M}_{k}$ is finitely 1-generated. In the context of self-similar fractals in the sense of John E. Hutchinson (cf. [3]) it will turn out to be convenient to endow $\partial \mathscr{M}$ with the cone topology $\mathscr{T}_{c}(\overline{\mathscr{M}})$. Let $(X, d)$ be a complete metric space with a left $\mathscr{M}$-action $\alpha: \mathscr{M} \longrightarrow C(X, X)$ by continuous maps. Such a presentation will be said to be contracting, if there exists a positive real number $\delta<1$ such that

$$
d(\alpha(s)(x), \alpha(s)(y)) \leq \delta \cdot d(x, y),
$$

for all $x, y \in X, s \in \mathscr{M}_{1}$ (cf. [3, § 2.2]). For such a metric space $(X, d)$ there exists a unique compact subset $K \subseteq X$ such that

(1) $K=\bigcup_{s \in \mathscr{M}_{1}} \alpha(s)(K)$,

(2) $K=\operatorname{cl}(\{\operatorname{Fix}(\alpha(t)) \mid t \in \mathscr{M}\} \subseteq X$.

Obviously, by definition every map $\alpha(t) \in C(X, X)$ is contracting, and thus has a unique fixed point $x_{t} \in X$. For short we call $K=K(\alpha) \subset X$ the attractor of the representation $\alpha$. One has the following (cf. Proposition 5.4).

Theorem C. Let $\mathscr{M}=\bigcup_{k \in \mathbb{N}_{0}} \mathscr{M}_{k}$ be a finitely 1-generated $\mathbb{N}_{0}$-graded monoid, let $(X, d)$ be a compact metric space and let $\alpha: \mathscr{M} \longrightarrow C(X, X)$ be a contracting representation of $\mathscr{M}$. Then for any point $x \in X, \alpha$ induces $a$ continuous map

$$
\kappa_{x}: \partial \mathscr{M} \longrightarrow K(\alpha)
$$


Moreover, if $\mathscr{M}$ is $\mathscr{T}$-regular, then $\kappa_{x}$ is surjective.

Under the general hypothesis of Theorem $\mathrm{C}$ we do not know whether the topological space $\left(\partial \mathscr{M}, \mathscr{T}_{c}(\overline{\mathscr{M}})\right)$ is necessarily compact (see Question 3). However, in case that it is compact, we call $\left(\partial \mathscr{M}, \mathscr{T}_{c}(\overline{\mathscr{M}})\right)$ universal attractor of the finitely 1-generated $\mathbb{N}_{0}$-graded $\mathscr{T}$-regular monoid $\mathscr{M}$.

Remark 1.2. Let $\mathscr{M}$ be a finitely 1 -generated monoid. Then $\partial \mathscr{M}$ carries canonically a probability measure $\mu_{\mathscr{M}}$ (cf. §4.5). Thus, by Theorem C, the attractor of the $\mathscr{M}$-fractal $((X, d), \alpha)$ carries the contact probability measure $\mu_{x}=\mu_{\circ}^{\kappa_{x}}$ for every point $x \in X$, which is given by

$$
\mu_{x}(B)=\mu_{\mathscr{M}}\left(\kappa_{x}^{-1}(B)\right), \quad B \in \operatorname{Bor}(K) .
$$

By (1), the monoid $\mathscr{M}$ is acting on $K$, and thus also on $L^{2}\left(K, \mathbb{C}, \mu_{x}\right)$ by bounded linear operators $\gamma(\omega)$, $\omega \in \mathscr{M}$ (cf. § 5.2) This defines a $C^{\star}$-algebra (cf. § 5.2)

$$
C^{\star}\left(\mathscr{M}, X, d, \mu_{x}\right)=\left\langle\gamma(\omega), \gamma(\omega)^{\star} \mid \omega \in \mathscr{M}\right\rangle \subseteq \mathcal{B}\left(L^{2}(K, \mathbb{C}, \mu)\right) .
$$

In case that the equivalence relation $\hat{\sim}$ generated by $\preceq$ on $\partial \mathscr{M}$ is different from $\approx$ (cf. (1.1)) the canonical map $\tilde{j}: \partial \mathscr{M} \rightarrow \partial \mathscr{M} / \hat{\sim}$ is not the identity.

Question 2. Does there exist a finitely 1-generated $\tau$-regular monoid $\mathscr{M}$ for which the map $\tilde{j}$ is not the identity, and an $\mathscr{M}$-fractal $((X, d), \alpha)$ such that $C^{\star}\left(\mathscr{M}, X, d \mu_{X}\right)$ is not isomorphic to $\partial C_{\lambda}(\mathscr{M})$ ?

\section{Posets and their boundaries}

A poset (or partially ordered set) is a set $X$ together with a reflexive and transitive relation $\preceq: X \times X \rightarrow\{t, f\}$ with the property that for all $x, y \in X$ satisfying $x \preceq y$ and $y \preceq x$ follows that $x=y$. By $\mathbb{N}=\{1,2, \ldots\}$ we denote the set of positive integers, and by $\mathbb{N}_{0}=\{0,1,2, \ldots\}$ we denote the set of non-negative integers, i.e., $\mathbb{N}_{0}$ is a commutative monoid.

\subsection{Cones, cocones and intervalls}

For a poset $(X, \preceq)$ and $\tau, \omega \in X$ the set

$$
\mathrm{C}_{\omega}=\{x \in X \mid x \preceq \omega\}
$$

will be called the cone defined by $\omega$, and

$$
\mathrm{J}_{\tau}=\{y \in X \mid y \succeq \tau\}
$$

the cocone defined by $\tau$. For $\tau \preceq \omega$ the set

$$
[\tau, \omega]=\mathrm{J}_{\tau} \cap \mathrm{C}_{\omega}=\{x \in X \mid \tau \preceq x \preceq \omega\}
$$

is called the closed intervall from $\tau$ to $\omega$, i.e., $[\omega, \omega]=\{\omega\}$. The poset $(X, \preceq)$ is said to be noetherian, if $\operatorname{card}\left(\mathrm{J}_{\tau}\right)<\infty$ for all $\tau \in X$.

\subsection{Complete posets}

For a poset $(X, \preceq)$ let

$$
\mathscr{D}(\mathbb{N}, X, \preceq)=\{f \in \mathscr{F}(\mathbb{N}, X) \mid \forall n, m \in \mathbb{N}: n \leq m \Longrightarrow f(n) \succeq f(m)\}
$$


denote the set of decreasing functions which we will - if necessary - identify with the set of decreasing sequences. A poset $(X, \preceq)$ is said to be complete, if for all $f \in \mathscr{D}(\mathbb{N}, X, \preceq)$ there exists an element $z \in X$ such that

$\left(\mathrm{CP}_{1}\right) f(n) \succeq z$ for all $n \in \mathbb{N}$, and

$\left(\mathrm{CP}_{2}\right)$ if $y \in X$ satisfies $f(n) \succeq y$ for all $n \in \mathbb{N}$, then $z \succeq y$.

Note that - if it exists - $z \in X$ is the unique element satisfying (i) and (ii) for $f \in \mathscr{D}(\mathbb{N}, X, \preceq)$. As usually, $z=\min (f)$ is called the minimum of $f \in \mathscr{D}(\mathbb{N}, X, \preceq)$.

\subsection{The poset completion of a poset}

Let $(X, \preceq)$ be a poset. For $u, v \in \mathscr{D}(\mathbb{N}, X, \preceq)$ put

$$
u \preceq v \Longleftrightarrow \forall n \in \mathbb{N} \exists k_{n} \in \mathbb{N}: u\left(k_{n}\right) \preceq v(n),
$$

and put

$$
u \sim v \Longleftrightarrow\left[(u \preceq v \wedge v \preceq u) \vee\left(v \preceq u \wedge v=c_{m}, m=\min (u)\right)\right],
$$

where $c_{z} \in \mathscr{D}(\mathbb{N}, X, \preceq), z \in X$, is given by $c_{z}(n)=z$ for all $n \in \mathbb{N}$.

Let $\approx$ be the equivalence relation generated by $\sim$ and put $\bar{X}=\mathscr{D}(\mathbb{N}, X, \preceq) / \approx$. Then the following properties hold for $(\bar{X}, \preceq)$.

Proposition 2.1. Let $(X, \preceq)$ be a poset.

(a) The relation $\preceq$ defined by (2.5) is reflexive and transitive.

(b) For any strictly increasing function $\alpha: \mathbb{N} \rightarrow \mathbb{N}$ and $u \in \mathscr{D}(\mathbb{N}, X, \preceq)$ one has $u \approx u \circ \alpha$.

(c) Define for $[u],[v] \in \bar{X}$ that $[u] \preceq[v]$ if, and only if, $u \preceq v$. Then $(\bar{X}, \preceq)$ is a poset.

(d) $(\bar{X}, \preceq)$ is complete.

Proof. (a) The relation $\preceq$ is obviously reflexive. Let $u, v, w \in \mathscr{D}(\mathbb{N}, X, \preceq), u \preceq v, v \preceq w$. Then for all $n \in \mathbb{N}$ there exists $h_{n}, k_{n} \in \mathbb{N}$ such that $u\left(h_{n}\right) \preceq v\left(k_{n}\right) \preceq w(n)$. Thus, $u \preceq w$.

(b) Let $u \in \mathscr{D}(\mathbb{N}, X, \preceq)$ and let $\alpha: \mathbb{N} \rightarrow \mathbb{N}$ be a strictly increasing function. Let $m<n, m, n \in \mathbb{N}$. Since $\alpha$ is strictly increasing, $\alpha(m)<\alpha(n)$. Then there exist $m_{0}, n_{0} \in \mathbb{N}$ such that $m_{0} \leq \alpha(m)<\alpha(n) \leq n_{0}$. Then one has $u\left(m_{0}\right) \succeq u(\alpha(m)) \succeq u(\alpha(n)) \succeq u\left(n_{0}\right)$. Thus $u \preceq u \circ \alpha$ and $u \circ \alpha \preceq u$, proving that $u \approx u \circ \alpha$.

(c) Let $[u]$, $[v] \in \bar{X}$, $[u] \preceq[v]$ and $[v] \preceq[u]$. Then, by definition, $u \preceq v$ and $v \preceq u$, and thus $u \approx v$, i.e., $[u]=[v]$.

(d) Let $\left\{u_{k}\right\}_{k \in \mathbb{N}} \in \mathscr{D}(\mathbb{N}, \bar{X}, \preceq)$, i.e., $u_{k} \in \bar{X}$ for all $k \in \mathbb{N}$. Then one has $u_{1} \succeq u_{2} \succeq \ldots$ by definition. Since each $u_{k} \in \mathscr{D}(\mathbb{N}, X, \preceq)$, one has $u_{k}(n) \succeq u_{k}(m)$ for all $n \leq m, m, n \in \mathbb{N}$. We define $v \in \mathscr{D}(\mathbb{N}, X, \preceq)$ by $v(n)=u_{n}(n)$, $n \in \mathbb{N}$. Then $[v] \in \bar{X}$ is the minimum of $\left\{u_{k}\right\}_{k \in \mathbb{N}}$. This yields the claim.

Assigning every element $x \in X$ the equivalence class containing the constant function $c_{X} \in \mathscr{D}(\mathbb{N}, X, \preceq)$ yields a strictly decreasing mapping of posets $\iota_{X}: X \rightarrow \bar{X}$. From now on $(X, \preceq)$ will be considered as a sub-poset of $(\bar{X}, \preceq)$. The poset $(\bar{X}, \preceq)$ will be called the poset completion of $(X, \preceq)$. The following fact is straightforward.

Fact 2.2. The map $\iota_{X}$ is a bijection if, and only if, $(X, \preceq)$ is complete.

Example 2.1. Let $X=\mathbb{N} \sqcup\{\infty\}$ and define $n \preceq m$ if, and only if, $n \geq m$, where “»” denotes the natural order relation. Then the poset $(X, \preceq)$ is complete and $\bar{X}=X$.

\subsection{The universal boundary of a poset}

For a poset $(X, \preceq)$ the poset $\partial X=\bar{X} \backslash \operatorname{im}\left(i_{X}\right)$ will be called the universal boundary of the poset $(X, \preceq)$. From now on we use the notation $x \succ y$ as a short form for $x \succeq y$ and $x \neq y$. A function $f: \mathbb{N} \rightarrow X$ will be said to be strictly decreasing, if $f(n+1) \prec f(n)$ for all $n \in \mathbb{N}$. The following fact will turn out to be useful. 
Fact 2.3. Let $f \in \mathscr{D}(\mathbb{N}, X, \preceq)$ be a decreasing function such that $[f] \in \partial X$. Then there exists a strictly decreasing function $h \in \mathscr{D}(\mathbb{N}, X, \preceq)$ such that $f \approx h$, i.e., $[f]=[h]$.

Proof. By hypothesis, $J=\operatorname{im}(f)$ is an infinite set. In particular, the set $\Omega=\left\{\min \left(f^{-1}(\{j\}) \mid j \in J\right\}\right.$ is an infinite and unbounded subset of $\mathbb{N}$. Let $e: \mathbb{N} \rightarrow \Omega$ be the enumeration function of $\Omega$, i.e., $e(1)=\min (\Omega)$, and recursively one has $e(k+1)=\min (\Omega \backslash\{e(1), \ldots, e(k)\})$. Then, by construction, $h=f \circ e$ is strictly decreasing, and, by Proposition 2.1(b), one has $f \approx h$, and hence the claim.

Fact 2.4. Let $(X, \preceq)$ be a noetherian poset, and let $(\bar{X}, \preceq)$ be its completion. Then for all $\tau \in X$ one has $\supset_{\tau}(\bar{X}) \subseteq$ $X$. In particular, $\mathrm{J}_{\tau}(\bar{X})=\mathrm{J}_{\tau}(X)$, where the cocones are taken in the respective posets.

Example 2.2. Let $X=A \sqcup B$, where $A, B=\mathbb{Z}$ and define

$$
n \preceq m \Longleftrightarrow(((n, m \in A \vee n, m \in B) \wedge n \leq m) \vee(n \in A \wedge m \in B)),
$$

where "s" denotes the natural order relation on $\mathbb{Z}$. Then $(X, \preceq)$ is a poset and its completion is given by $\bar{X}=$ $\mathbb{Z} \sqcup\{-\infty\} \sqcup \mathbb{Z} \sqcup\{-\infty\}$. For $n \in A$, one has $\supset_{n}(X) \neq \supset_{n}(\bar{X})$, since $-\infty \in B$ is in $\supset_{n}(\bar{X})$, but not in $\supset_{n}(X)$.

\subsection{The cone topology}

Let $(X, \preceq)$ be a poset, and let $(\bar{X}, \preceq)$ denote its completion. For $\tau$, $\omega \in X$ let

$$
S(\tau, \omega)=\{x \in X \mid x \preceq \tau \wedge x \preceq \omega\} .
$$

By transitivity,

$$
\mathrm{C}_{\tau}(\bar{X}) \cap \mathrm{C}_{\omega}(\bar{X})=\bigcup_{z \in S(\tau, \omega)} \mathrm{C}_{z}(\bar{X}) .
$$

In particular,

$$
\mathscr{B}_{c}(\bar{X})=\{\{x\} \mid x \in X\} \cup\left\{\mathrm{C}_{\omega}(\bar{X}) \mid \omega \in X\right\}
$$

is a base of a topology $\mathscr{T}_{c}(\bar{X})$ - the cone topology - on $\bar{X}$. By construction, the subspace $X$ is discrete and open, and the subspace $\partial X$ is closed.

For $\omega \in \bar{X}$ let $\mathscr{N}_{c}(\omega)$ denote the set of all open neighborhoods of $\omega$ with respect to the cone-topology, and put $\mathscr{S}(\omega)=\bigcap_{U \in \mathscr{N}_{c}(\omega)} U$. Then, by construction, one has $\mathscr{S}(\omega)=\{\omega\}$ for $\omega \in X$, and $\mathscr{S}(\omega)=\mathrm{C}_{\omega}(\bar{X})$ for $\omega \in \partial X$. This implies the following.

Proposition 2.5. Let $(X, \preceq)$ be a poset, and let $(\bar{X}, \preceq)$ denote its completion. Then $\left(\bar{X}, \mathscr{T}_{c}(\bar{X})\right.$ ) is a $T_{0}$-space (or Kolmogorov space).

Proof. Let $\tau, \omega \in \bar{X}, \tau \neq \omega$. If either $\tau \in X$ or $\omega \in X$, then either $\{\tau\}$ or $\{\omega\}$ is an open set. So we may assume that $\tau, \omega \in \partial X$. As $\mathscr{S}(\omega)=\mathrm{C}_{\omega}(\bar{X})$, either there exists $U \in \mathscr{N}_{c}(\omega), \tau \notin U$, or $\tau \preceq \omega$. By changing the role of $\omega$ and $\tau$, either there exists $V \in \mathscr{N}_{c}(\tau), \omega \notin V$, or $\omega \preceq \tau$. Since $\tau \preceq \omega$ and $\omega \preceq \tau$ is impossible, this yields the claim.

\subsection{The fine topology}

For a partially ordered set $(X, \preceq)$ let

$$
\mathscr{S}=\left\{\{\tau\}, C_{\tau}(\bar{X}), C_{\tau}(\bar{X})^{C} \mid \tau \in X\right\}
$$

denote the set of all subsets of $X$ of cardinality 1, all cones and their complements in $X$. Then $\mathscr{S}$ is a subbasis of a topology $\mathscr{T}_{f}(\bar{X})$ on $\bar{X}$ which we will call the fine topology on $\bar{X}$. In particular, the set $\Omega=\left\{X=\bigcap_{1 \leq j \leq r} X_{j} \mid\right.$ $\left.X_{1}, \ldots, X_{r} \in \mathscr{S}\right\}$ is a base of the topology $\mathscr{T}_{f}(\bar{X})$. By definition, this topology has the following properties. 
Fact 2.6. Let $(X, \preceq)$ be a partially ordered set. Then

(a) $\left(\bar{X}, \mathscr{T}_{f}(\bar{X})\right)$ is a $T_{2}$-space (or Hausdorff space).

(b) $\mathscr{T}_{c}(\bar{X}) \subseteq \mathscr{T}_{f}(\bar{X})$.

\subsection{The $\sim$-boundary}

There is another type of boundary for a poset, the $\sim$-boundary, which seems to be relevant for the study of fractals (see (5.6)). Let $(X, \preceq)$ be a noetherian poset, and let $(\bar{X}, \preceq)$ denote its completion. Put

$$
\Omega=\Delta(X) \sqcup\{(\varepsilon, \eta) \in \partial X \times \partial X \mid \varepsilon \preceq \eta\},
$$

where $\Delta(X)=\{(x, x) \mid x \in X\}$, and let $\hat{\sim}$ denote the equivalence relation on $\bar{X}$ generated by the relation $\Omega$. Then one has a canonical map

$$
\pi: \bar{X} \rightarrow \widetilde{X},
$$

where $\widetilde{X}=\bar{X} / \hat{\sim}$. By construction, $\left.\pi\right|_{X}$ is injective. The set $\widetilde{\partial} X=\widetilde{X} \backslash \pi(X)$ will be called the $\sim$-boundary of the poset $(X, \preceq)$. We put

$$
I(\sim)=\{(\omega, \tau) \in \bar{X} \times \bar{X} \mid \omega \hat{\sim} \tau\} \subseteq \bar{X} \times \bar{X}
$$

The set $\widetilde{X}$ carries the quotient topology $\mathscr{T}_{q}(\widetilde{X})$ with respect to the mapping $\pi$ and the topological space $\left(\bar{X}, \mathscr{T}_{C}(\bar{X})\right)$. In particular, the subspace $\pi(X) \subseteq \widetilde{X}$ is discrete and open, and $\widetilde{\partial} X \subseteq \widetilde{X}$ is closed. For $\omega \in \bar{X}$ we put $\widetilde{\mathrm{C}}_{\omega}=\pi\left(\mathrm{C}_{\omega}(\bar{X})\right)$. The space $\widetilde{X}$ will be considered merely as topological space. It has the following property.

Proposition 2.7. The topological space $\left(\widetilde{X}, \mathscr{T}_{q}(\widetilde{X})\right)$ is a $T_{1}$-space.

Proof. For $\omega \in \bar{X}$ one has

$$
\mathscr{S}(\pi(\omega))=\pi\left(\bigcap_{\tau \hat{\sim} \omega} \mathscr{S}(\tau)\right)=\pi\left(\bigcap_{\tau \hat{\sim} \omega} \mathrm{C}_{\tau}(\bar{X})\right)=\{\pi(\omega)\}
$$

This yields the claim.

\section{Monoids and their boundaries}

A monoid (or semigroup with unit) $\mathscr{M}$ is a set with an associative multiplication _- ${ }_{-}: \mathscr{M} \times \mathscr{M} \rightarrow \mathscr{M}$ and a distinguished element $1 \in \mathscr{M}$ satisfying $1 \cdot x=x \cdot 1=x$ for all $x \in \mathscr{M}$. For a monoid $\mathscr{M}$ we denote by

$$
\mathscr{M}^{\times}=\{x \in \mathscr{M} \mid \exists y \in \mathscr{M}: x \cdot y=y \cdot x=1\}
$$

the maximal subgroup contained in $\mathscr{M}$.

\section{1 $\mathbb{N}_{0}$-graded monoids}

The set of non-negative integers $\mathbb{N}_{0}=\{0,1,2, \ldots\}$ together with addition is a monoid. A monoid $\mathscr{M}$ together with a homomorphism of monoids $|-|: \mathscr{M} \rightarrow \mathbb{N}_{0}$ is called an $\mathbb{N}_{0}$-graded monoid. For $k \in \mathbb{N}_{0}$ one defines $\mathscr{M}_{k}=\{x \in \mathscr{M}|| x \mid=k\}$. The $\mathbb{N}_{0}$-graded monoid $\mathscr{M}$ is said to be connected, if $\mathscr{M}_{0}=\{1\}$. One has the following straightforward fact.

Fact 3.1. For a connected $\mathbb{N}_{0}$-graded monoid $\mathscr{M}$ one has $\mathscr{M}^{\times}=\{1\}$.

If $\mathscr{Q}$ and $\mathscr{M}$ are $\mathbb{N}_{0}$-graded monoids, a homomorphism $\phi: \mathscr{Q} \rightarrow \mathscr{M}$ is a homomorphism of $\mathbb{N}_{0}$-graded monoids, if $\phi\left(\mathscr{Q}_{k}\right) \subseteq \mathscr{M}_{k}$ for all $k \in \mathbb{N}_{0}$. The following property is straightforward. 
Proposition 3.2. Let $\phi: \mathscr{Q} \rightarrow \mathscr{M}$ be a homomorphism of $\mathbb{N}_{0}$-graded monoids. Then $\phi$ is monotone, i.e., $x, y \in$ $\mathscr{Q}, x \preceq y$ implies $\phi(x) \preceq \phi(y)$, and thus induces a monotone map

$$
\mathscr{D} \phi: \mathscr{D}(\mathbb{N}, \mathscr{Q}, \preceq) \longrightarrow \mathscr{D}(\mathbb{N}, \mathscr{M}, \preceq) .
$$

Let $\bar{\phi}: \overline{\mathscr{Q}} \rightarrow \overline{\mathscr{M}}$ denote the induced map. Let $\partial \bar{\phi}: \partial \mathscr{Q} \longrightarrow \partial \mathscr{M}$ be the map induced by $\bar{\phi}$. Then $\partial \bar{\phi}$ is continuous with repect to the cone topology.

Proof. Let $\tau \in \mathscr{M}$. Then the monotony of $\mathscr{D} \phi$ implies that

$$
\bar{\phi}^{-1}\left(C_{\tau}(\overline{\mathscr{M}})\right)=\bigcup_{y \in \mathscr{S}} C_{y}(\overline{\mathcal{Q}}),
$$

where $\mathscr{S}=\left\{q \in \overline{\mathscr{Q}} \mid \bar{\phi}(q) \in C_{\tau}(\overline{\mathscr{M}})\right\}$. Thus $\bar{\phi}$ and $\partial \phi$ are continuous.

\subsection{1-generated monoids}

For any set $Y$ there exists a free monoid $\mathscr{F}\langle Y\rangle$ which is naturally $\mathbb{N}_{0}$-graded. Moreover, $\mathscr{F}\langle Y\rangle$ is connected and $\mathscr{F}\langle Y\rangle_{1}=Y$. For an $\mathbb{N}_{0}$-graded monoid $\mathscr{M}$ there exists a canonical homomorphism of $\mathbb{N}_{0}$-graded monoids

$$
\phi_{\mathscr{M}}: \mathscr{F}\left\langle\mathscr{M}_{1}\right\rangle \longrightarrow \mathscr{M}
$$

satisfying $\phi_{\mathscr{M}, 1}=\operatorname{id} \mathscr{M}_{1}$. The $\mathbb{N}_{0}$-graded monoid $\mathscr{M}$ is said to be 1-generated, if $\phi_{\mathscr{M}}$ is surjective. In particular, such a monoid is connected. By definition, free monoids are 1-generated. Moreover, $\mathscr{M}$ is said to be finitely 1-generated, if it is 1-generated and $\mathscr{M}_{1}$ is a finite set. The following important question remains unanswered in this paper.

Question 3. Does there exist a finitely 1-generated monoid $\mathscr{M}$ satisfying $\mathscr{T}_{c}(\overline{\mathscr{M}}) \neq \mathscr{T}_{f}(\overline{\mathscr{M}})$ ?

\subsection{Monoids as posets}

Let $\mathscr{M}$ be a monoid. For $x \in \mathscr{M}$, put

$$
\begin{aligned}
& \mathscr{M} x=\{y x \mid y \in \mathscr{M}\} ; \\
& x \mathscr{M}=\{x y \mid y \in \mathscr{M}\} .
\end{aligned}
$$

For $x, y \in \mathscr{M}$ we define

$$
x \preceq y \Longleftrightarrow x \mathscr{M} \subseteq y \mathscr{M},
$$

i.e., $x \preceq y$ if, and only if, there exists $z \in \mathscr{M}$ such that $x=y z$.

\subsection{Left cancellative monoids}

A monoid $\mathscr{M}$ is said to be left cancellative if $x y=x z$ implies $y=z$ for all $x, y, z \in \mathscr{M}$; and right cancellative if $y x=z x$ implies $y=z$ for all $x, y, z \in \mathscr{M}$.

Proposition 3.3. Let $\mathscr{M}$ be a left-cancellative monoid. For $x, y \in \mathscr{M}$ one has $\mathscr{M} x=\mathscr{M} y$ if, and only if, there exists $z \in \mathscr{M}^{\times}$such that $y=x z$.

Proof. For $z \in \mathscr{M}^{\times}$one has $z \mathscr{M}=\mathscr{M}$. Thus for $x \in \mathscr{M}$ and $y=x z$, multiplying by $x$ from the left yields $y \mathscr{M}=x \mathscr{M}$. Viceversa, suppose $x \mathscr{M}=y \mathscr{M}$ for $x, y$ in $\mathscr{M}$. Then there exist $z, w \in \mathscr{M}$ such that $y=x z$ and $x=y w$. Hence $y=y w z$ and $x=x z w$. Thus left cancellation implies $z w=1=w z$, showing that $z, w \in \mathscr{M}^{\times}$. 
Corollary 3.4. Let $\mathscr{M}$ be a left-cancellative monoid. Then $\left(\mathscr{M} \mid \mathscr{M}^{\times}, \preceq\right)$ is a poset.

Remark 3.5. If left cancellation is replaced by right cancellation, then one has $x \mathscr{M}=y \mathscr{M}$ if, and only if, there exists $z \in \mathscr{M}^{\times}$such that $y=z x$.

\subsection{1-generated monoids as posets}

Proposition 3.6. Let $\mathscr{M}$ be a connected $\mathbb{N}_{0}$-graded monoid. For $x, y \in \mathscr{M}$ one has $x \mathscr{M}=y \mathscr{M}$ if, and only if, $x=y$.

Proof. Suppose $x \mathscr{M}=y \mathscr{M}$, for $x, y \in \mathscr{M}$. Then there exist $z, w \in \mathscr{M}$ such that $x=y z$ and $y=x w$, so $|x|=|y|+|z|$ and $|y|=|x|+|w|$. Thus $|z|=0=|w|$. Since $\mathscr{M}$ is connected, this implies $z=1=w$.

As a consequence one obtains the following.

Corollary 3.7. Let $\mathscr{M}$ be a 1-generated $\mathbb{N}_{0}$-graded monoid. Then $(\mathscr{M}, \preceq)$ is a poset. If $\mathscr{M}$ be finitely 1-generated, then $(\mathscr{M}, \preceq)$ is a noetherian poset.

Remark 3.8. The following example shows that the universal boundary $\partial \mathscr{M}$ is in general different from the $\sim$-boundary $\widetilde{\partial} \mathscr{M}$. Let $\mathscr{M}=\langle x, y, z \mid x z=z x\rangle$. Consider

$$
\begin{array}{ll}
f_{1}: \mathbb{N} \rightarrow M, & f_{1}(n)=(x z)^{n}, \\
f_{2}: \mathbb{N} \rightarrow M, & f_{2}(n)=x^{n}, \\
f_{3}: \mathbb{N} \rightarrow M, & f_{3}(n)=z^{n} .
\end{array}
$$

Then $f_{2} \succeq f_{1} \preceq f_{3}$. Hence $\pi\left(f_{1}\right)=\pi\left(f_{2}\right)=\pi\left(f_{3}\right) \in \widetilde{\partial} \mathscr{M}$, and $\pi: \partial M \rightarrow \widetilde{\partial} \mathscr{M}$ is not injective.

\subsection{Abelian semigroups generated by idempotents}

Let $E$ be an abelian semigroup being generated by a set of elements $\Sigma \subseteq E$ satisfying $\sigma^{2}=\sigma$ for all $\sigma \in \Sigma$, i.e., all elements of $\Sigma$ are idempotents. Then every element $u \in E$ is an idempotent, and one may define a partial order " $\preceq$ " on $E$ by

$$
u \preceq v \quad \Longleftrightarrow \quad u \cdot v=v,
$$

for $u, v \in E$. Let $\mathscr{R}=\{(u, v) \in \Sigma \times \Sigma \mid u \preceq v\}$. By definition, one has

$$
E=\left\{u=\sigma_{1} \cdots \sigma_{r} \mid \sigma_{i} \in \Sigma\right\} .
$$

Hence

$$
E \simeq \mathscr{F}^{\mathrm{ab}}(\Sigma) / R,
$$

where $\mathscr{F}^{\mathrm{ab}}(\Sigma)$ is the free abelian semigroup over the set $\Sigma$, and $R$ is the relation

$$
R=\{(u v, v) \mid(u, v) \in \mathscr{R}\} \subseteq \mathscr{F}^{\mathrm{ab}}(\Sigma) \times \mathscr{F}^{\mathrm{ab}}(\Sigma),
$$

i.e., $E=\mathscr{F}^{\mathrm{ab}}(\Sigma) / R^{\sim}$, where $R^{\sim}$ is the equivalence relation on $\mathscr{F}^{\mathrm{ab}}(\Sigma)$ generated by the set $R$. Let

$$
\widehat{E}=\{\chi: E \rightarrow\{0,1\} \mid \chi \text { a semigroup homomorphism, } \chi(0)=0, \chi \not \equiv 0\}
$$

Then $\widehat{E}$ coincides with the set of characters of the $C^{\star}$-algebra $C^{\star}(E)$ generated by $E$ (satisfying $e^{\star}=e$ for all $e \in E$ ), and hence carries naturally the structure of a compact topological space. By construction, $\widehat{E}$ can be identified with a subset of $\mathcal{F}(\Sigma,\{0,1\})$ - the set of functions from $\Sigma$ to $\{0,1\}$. In more detail,

$$
\widehat{E}=\{\phi \in \mathcal{F}(\Sigma,\{0,1\}) \mid \forall(u, v) \in \mathscr{R}: \phi(v)=\phi(u) \cdot \phi(v)\}
$$

Thus identifying $\mathscr{F}(\Sigma,\{0,1\})$ with $\{0,1\}^{\Sigma}$, one obtains that

$$
\widehat{E}=\left\{\left(\eta_{\sigma}\right)_{\sigma \in \Sigma} \in\{0,1\}^{\Sigma} \mid \forall(u, v) \in \mathscr{R}: \sigma_{v}=\sigma_{u} \cdot \sigma_{v}\right\} .
$$




\subsection{The semigroup of idempotents generated by a set of subsets of a set}

Let $X$ be a set, and let $S \subseteq \mathscr{P}(X)$ be a set of subsets of $X$. Then $S$ generates an algebra of sets $\mathscr{A}(S) \subseteq \mathscr{P}(X)$, i.e., the sets of $\mathscr{A}(S)$ consist of the finite intersections of sets in $S$. Then

$$
E(S)=\left\langle I_{A} \mid A \in \mathscr{A}(S)\right\rangle \subseteq \mathscr{F}(X,\{0,1\})
$$

is an abelian semigroup being generated by the set of idempotents

$$
\Sigma=\left\{I_{Y} \mid Y \in S\right\} .
$$

Moreover, by (3.14), one has

$$
\widehat{E}(S)=\{\phi \in \mathcal{F}(S,\{0,1\}) \mid \forall U, V \in S, V \subseteq U: \phi(V)=\phi(U) \cdot \phi(V)\}
$$

\subsection{The Laca-boundary of a monoid}

Let $\mathscr{M}$ be a 1-generated monoid. Then one chooses

$$
S=\{\omega \cdot \mathscr{M} \mid \omega \in \mathscr{M}\}
$$

to consist of all principal right ideals. For short we call the compact set $ð \mathscr{M}=\widehat{E}(S)$ for $S$ as in (3.19) the Laca boundary of $\mathscr{M}$. For an infinite word $\underline{\omega}=\left(\omega_{k}\right) \in \mathscr{D}(\mathbb{N}, \mathscr{M}, \succeq)$ and for $\tau \in \mathscr{M}$ one defines the element $\chi_{\underline{\omega}} \in \widehat{E}(S)$ by $\chi_{\underline{\omega}}(\tau \mathscr{M})=1$ if, and only if, there exists $k \in \mathbb{N}$ such that $\omega_{k} \in \tau \mathscr{M}$, i.e., $\tau \succeq \omega_{k}$, and thus $\tau \succeq \underline{\omega}$. This yields a map

$$
\chi \cdot: \mathscr{D}(\mathbb{N}, \mathscr{M}, \preceq) \longrightarrow \widehat{E}(S)
$$

(cf. [5, § 2.2]). By definition, it has the following property:

Proposition 3.9. For $\underline{\omega}=\left(\omega_{k}\right) \in \mathscr{D}(\mathbb{N}, \mathscr{M}, \preceq), \tau \in \mathscr{M}$, one has $\chi_{\underline{\omega}}(\tau \mathscr{M})=1$ if, and only if, $\tau \succeq \underline{\omega}$. In particular, one has $\chi_{\eta}=\chi_{\underline{\omega}}$ if, and only if, $\underline{\eta} \approx \underline{\omega}$, and hence $\chi$. induces an injective map

$$
\bar{\chi} . \partial \mathscr{M} \longrightarrow \partial \mathscr{M} .
$$

Proof. The first part has already been established before. Let $\underline{\eta}=\left(\eta_{k}\right)$. Then by the first part, $\underline{\omega} \succeq \underline{\eta}$ implies that for all $\tau \in \mathscr{M}$ one has

$$
\chi \underline{\omega}(\tau \mathscr{M})=1 \Longrightarrow \chi_{\underline{\eta}}(\tau \mathscr{M})=1 .
$$

Thus as $\operatorname{im}\left(\chi_{\omega}\right) \subseteq\{0,1\}$ one concludes that $\underline{\omega} \succeq \eta$ and $\underline{\omega} \preceq \eta$ implies that $\chi_{\underline{\omega}}=\chi_{\eta}$. On the other hand $\chi_{\underline{\eta}}=\chi_{\underline{\omega}}$ implies that $1=\chi_{\underline{\eta}}\left(\eta_{k} \mathscr{M}\right)=\chi_{\underline{\omega}}\left(\eta_{k} \mathscr{M}\right)$ for all $\bar{k} \in \mathbb{N}$. In particular, $\underline{\eta} \succeq \underline{\omega}$. Interchanging the roles of $\underline{\eta}$ and $\underline{\omega}$ yields $\underline{\omega} \succeq \underline{\eta}$, and thus $\underline{\eta} \approx \underline{\omega}$ (cf. section 2.3). The last part is a direct consequence of the definition of д $\mathscr{M}$.

The following theorem shows that for a 1-generated $\mathbb{N}_{0}$-graded monoid $\mathscr{M}$ its universal boundary $\partial \mathscr{M}$ with the fine topology is a totally-disconnected compact space.

Theorem 3.10. The map $\bar{\chi} .:\left(\partial \mathscr{M}, \mathscr{T}_{f}(\overline{\mathscr{M}})\right) \longrightarrow \partial \mathscr{M}$ is a homeomorphism.

Proof. It is well known that $\chi$ is surjective (cf. [5, Lemma 2.3]), and thus $\bar{\chi}$ is surjective. By Proposition 3.9, $\bar{\chi}$ is injective, and hence $\bar{\chi}$ is a bijection. The sets

$$
U_{\tau}^{\varepsilon}=\{\eta \in \widehat{E}(\mathscr{M}) \mid \eta(\tau \mathscr{M})=\varepsilon\}, \quad \tau \in \mathscr{M}, \quad \varepsilon \in\{0,1\}
$$

form a subbasis of the topology of $\widehat{E}(\mathscr{M})$, and

$$
\bar{\chi}^{-1}\left(U_{\tau}^{1}\right)=C_{\tau}(\overline{\mathscr{M}}) \cap \partial \mathscr{M}
$$

by (3.22). Hence $\bar{\chi}^{-1}\left(U_{\tau}^{0}\right)=C_{\tau}(\overline{\mathscr{M}})^{C} \cap \partial \mathscr{M}$ and this yields the claim. 
The proof of Theorem 3.10 has also shown that

$$
\bar{\chi}^{-1}: \partial \mathscr{M} \longrightarrow\left(\partial \mathscr{M}, \mathscr{T}_{C}(\overline{\mathscr{M}})\right)
$$

is a bijective and continuous map. Thus, if $\left(\partial \mathscr{M}, \mathscr{T}_{c}(\overline{\mathscr{M}})\right.$ ) is Hausdorff, then $\bar{\chi}^{-1}$ is a homeomorphism (cf. [1, $\S 9.4$, Corollary 2]). This has the following consequence.

Proposition 3.11. Let $\mathscr{M}$ be a 1-generated $\mathbb{N}_{0}$-graded monoid such that $\left(\partial \mathscr{M}, \mathscr{T}_{c}(\overline{\mathscr{M}})\right)$ is Hausdorff. Then $\mathscr{M}$ is $\mathscr{T}$-regular.

In contrast to Proposition 3.2 one has the following property for the Laca boundary of monoids.

Proposition 3.12. Let $\phi: \mathscr{Q} \longrightarrow \mathscr{M}$ be a surjective homomorphism of connected $\mathbb{N}_{0}$-graded monoids. Then $\phi$ induces an injective continuous map $\phi_{\Im}: ð \mathscr{M} \longrightarrow ð \mathscr{Q}$.

Proof. By Proposition 3.6, $\phi$ induces a map $\phi_{\Sigma}: \Sigma(\mathscr{Q}) \rightarrow \Sigma(\mathscr{M})$ given by

$$
\phi_{\Sigma}(\omega \mathscr{Q})=\phi(\omega) \mathscr{M} .
$$

Moreover, for $x, y \in \mathscr{Q}$ one has $x \preceq y$, if and only if, $x \mathscr{Q} \subseteq y \mathscr{Q}$, if and only if there exists $z \in \mathscr{Q}$ such that $x=y \cdot z$. From the last statement one concludes that $\phi_{\Sigma}(x \mathscr{Q}) \subseteq \phi_{\Sigma}(z \mathscr{Q})$. Thus, by (3.11), $\phi_{\Sigma}$ induces a homomorphism of semigroups

$$
\phi_{E}: E(\mathscr{Q}) \longrightarrow E(\mathscr{M})
$$

and thus a map

$$
\phi_{\widehat{E}}^{\circ}: \widehat{E}(\mathscr{M}) \cup\{0\} \longrightarrow \widehat{E}(\mathscr{Q}) \cup\{0\} .
$$

If $\phi$ is surjective, then $\phi_{E}$ is surjective, and $\phi_{\widehat{E}}^{\circ}$ restricts to a map

$$
\phi_{\widehat{E}}: \widehat{E}(\mathscr{M}) \longrightarrow \widehat{E}(\mathscr{Q})
$$

It is straightforward to verify that $\phi_{\widehat{E}}$ is continuous and injective.

\section{Free monoids and trees}

Let $\mathscr{F}_{n}=\mathscr{F}\left\langle x_{1}, \ldots, x_{n}\right\rangle$ be the free monoid on $n$ generators. Let $S=\left\{x_{1}, \ldots, x_{n}\right\}$ be the set of generators, and let $\left|\_\right|: \mathscr{F}_{n} \rightarrow \mathbb{N}_{0}$ be the grading morphisms, i.e., $|y|=1$ if and only if $y \in S$. The Cayley graph $\Gamma\left(\mathscr{F}_{n}, S\right)$ of $\mathscr{F}_{n}$ with respect to $S$ is the graph defined by

$$
\begin{aligned}
& V=\left\{x \mid x \in \mathscr{F}_{n}\right\} \\
& E=\left\{\left(x, x x_{i}\right) \in V \times V \mid x \in \mathscr{F}_{n}, x_{i} \in S\right\} .
\end{aligned}
$$

The origin and terminus maps $o, t: E \rightarrow V$ are given by the projection onto the first and second coordinate, respectively. Then $\Gamma\left(\mathscr{F}_{n}, S\right)$ is an $n$-regular tree with root 1 and all edges pointing away from 1 . The graph $\Gamma\left(\mathscr{F}_{n}, S\right)$ coincides with an orientation of the $n$-regular tree $T_{n}$.

\subsection{The boundary of the $n$-regular tree}

The boundary $\partial T_{n}$ of $T_{n}$ is the set of equivalence classes of infinite paths without backtracking under the relation $\sim$ defined by the shift, i.e.

$$
v_{0} v_{1} v_{2} \cdots \sim v_{1} v_{2} v_{3} \ldots
$$


We denote by $[v, w)$ the unique path starting at $v$ in the class $\omega$ and define

$$
I_{V}=\left\{\omega \in \partial T_{n} \mid v \in[1, \omega)\right\}
$$

the interval of $\partial T_{n}$ starting at $v$. Then $\partial T_{n}$ is compact with respect to the topology $\mathscr{T}_{I}$ generated by $\left\{I_{v}\right\}_{v \in V}$.

For any $[\rho] \in \partial T_{n}$ there exists a unique ray $\rho=\left(e_{k}\right)_{k \in \mathbb{N}}, o(\rho)=o\left(e_{1}\right)=1$. One can assign to $\rho$ the decreasing function $\omega_{\rho} \in \mathscr{D}\left(\mathbb{N}, \mathscr{F}_{n}, \preceq\right)$ given by $\omega_{\rho}(k)=t\left(e_{k}\right)$. The map $\varphi: \partial T_{n} \rightarrow \partial \mathscr{F}_{n}$ given by

$$
\varphi([\rho])=\left[\omega_{\rho}\right]
$$

is a bijection. Hence one can identify $\partial T_{n}$ with $\partial \mathscr{F}_{n}$.

\subsection{The space $\left(\overline{\mathscr{F}}_{n}, \mathscr{T}_{c}\left(\overline{\mathscr{F}}_{n}\right)\right)$}

Every cone $C_{\tau}\left(\overline{\mathscr{F}}_{n}\right)$ defines a rooted subtree $T_{\tau}$ of $T_{n}$ satisfying $\partial T_{\tau}=\partial \mathscr{F}_{n} \cap C_{\tau}\left(\overline{\mathscr{F}}_{n}\right)$. Thus every covering $\bigcup_{\tau \in U} C_{\tau}\left(\overline{\mathscr{F}}_{n}\right) \cap \partial \mathscr{F}_{n}$ of the boundary of $\partial \mathscr{F}_{n}$ by cones defines a forest $F=\bigcup_{\tau \in U} T_{\tau}$. Let $F=\bigcup_{i \in I} F_{i}$ be the decomposition of $F$ in connected components. Then $\partial T_{n}=\partial F=\bigsqcup_{i \in I} \partial F_{i}$, where $\sqcup$ denotes disjoint union. Hence the compactness of $\partial T_{n}$ implies $|I|<\infty$.

As $\partial F_{i} \subseteq \partial T_{n}$ is closed, and hence compact, a similar argument shows that there exist finitely many cones $C_{\tau_{i, j}}, 1 \leq j \leq r_{i}$, such that $F_{i}=\bigcup_{1 \leq j \leq r_{i}} T_{\tau_{i, j}}$. Thus, if $\bigcup V$ is an open covering of $\overline{\mathscr{F}}_{n}$ by open sets, it can be refined to a covering $\bigcup U$, where $U$ consists either of a cone $C_{\tau}\left(\overline{\mathscr{F}}_{n}\right)$ or of a singleton set $\{\omega\}, \omega \in \mathscr{F}_{n}$. Let $\Lambda \subseteq T_{n}$ be the subtree being generated by the vertices $\tau_{i, j}$. Then $\Lambda$ is a finite subtree, and the only vertices of $T_{n}$ not being covered by $\left.\bigcup_{i, j} C_{\tau_{i, j}}\left(\overline{\mathscr{F}}_{n}\right)\right)$ are contained in $V(\Lambda)$. This shows that $\left(\overline{\mathscr{F}}_{n}, \mathscr{T}_{c}\left(\overline{\mathscr{F}}_{n}\right)\right)$ is a compact space.

\subsection{The space $\left(\overline{\mathscr{M}}, \mathscr{T}_{c}(\overline{\mathscr{M}})\right)$}

Let $\mathscr{M}$ be a $\mathscr{T}$-regular finitely 1 -generated monoid. Then, by definition, $\left(\partial \mathscr{M}, \mathscr{T}_{c}(\overline{\mathscr{M}})\right)$ is a Hausdorff space, and hence $\left(\overline{\mathscr{M}}, \mathscr{T}_{c}(\overline{\mathscr{M}})\right.$ ) is a Hausdorff space. By Proposition 3.2, the canonical mapping $\phi_{\mathscr{M}}: \mathscr{F} \rightarrow \mathscr{M}$ (cf. (3.4)) induces a continuous surjective map $\bar{\phi}_{\mathscr{M}}: \overline{\mathscr{F}} \rightarrow \overline{\mathscr{M}}$. This shows the following.

Proposition 4.1. Let $\mathscr{M}$ be a finitely 1 -generated $\mathbb{N}_{0}$-graded $\mathscr{T}$-regular monoid. Then $\left(\overline{\mathscr{M}}, \mathscr{T}_{c}(\tilde{\mathscr{M}})\right)$ is a compact space.

\subsection{The canonical probability measure on the boundary of a regular tree}

By Carathéodory’s extension theorem the assignment

$$
\mu\left(I_{v}\right)=n^{-|v|}
$$

defines a unique probability measure $\mu: \operatorname{Bor}\left(\partial T_{n}\right) \rightarrow \mathbb{R}_{0}^{+}$. Hence the corresponding probability measure $\mu: \operatorname{Bor}\left(\partial \mathscr{F}_{n}\right) \rightarrow \mathbb{R}_{0}^{+}$satisfies

$$
\mu\left(\partial \mathscr{F}_{n} \cap C_{\tau}\left(\overline{\mathscr{F}}_{n}\right)\right)=n^{-|\tau|} \text { for } \tau \in \mathscr{F}_{n} .
$$

Definition. Let_._: $\mathscr{F}_{n} \times \partial \mathscr{F}_{n} \rightarrow \partial \mathscr{F}_{n}$ be the map given by

$$
x \cdot[\omega]=[x \omega],
$$

where $x \omega: \mathbb{N} \rightarrow \mathscr{F}_{n}$ is given by $(x \omega)(n)=x \omega(n)$

Note that this action is well defined, since $\omega \sim \omega^{\prime}$ implies that $x \omega \sim x \omega^{\prime}$. 
Definition. Let _. _: $L^{2}\left(\partial \mathscr{F}_{n}, \mathbb{C}, \mu\right) \times \mathscr{F}_{n} \rightarrow L^{2}\left(\partial \mathscr{F}_{n}, \mathbb{C}, \mu\right)$ be the map given by

$$
f . x={ }^{x} f,
$$

where

$$
\left({ }^{x} f\right)([\omega])=f([x \omega]) .
$$

Note that for $f \in L^{2}\left(\partial \mathscr{F}_{n}, \mathbb{C}, \mu\right)$ one has ${ }^{x} f \in L^{2}\left(\partial \mathscr{F}_{n}, \mathbb{C}, \mu\right)$, since

$$
\begin{aligned}
\left\|{ }^{x} f\right\|_{2}^{2} & =\int_{\partial T_{n}}\left|{ }^{x} f\right|^{2} d \mu \\
& =\int_{x \partial T_{n}}|f|^{2} d \mu \\
& \leq \int_{\partial T_{n}}|f|^{2} d \mu \\
& =\|f\|_{2}^{2},
\end{aligned}
$$

where (4.13) follows since $x \partial \mathscr{F}_{n} \subseteq \partial \mathscr{F}_{n}$.

Definition. For $z \in \mathscr{F}_{n}$ we define the map $T_{z}: L^{2}\left(\partial \mathscr{F}_{n}, \mathbb{C}, \mu\right) \rightarrow L^{2}\left(\partial \mathscr{F}_{n}, \mathbb{C}, \mu\right)$ by

$$
T_{z}(f)={ }^{z} f .
$$

Fact 4.2. $\mathscr{F}_{n}$ acts via $T$. on $L^{2}\left(\partial \mathscr{F}_{n}, \mathbb{C}, \mu\right)$ by bounded linear operators.

Proof. Let $z \in \mathscr{F}_{n}$. For $f, g \in L^{2}\left(\partial \mathscr{F}_{n}, \mathbb{C}, \mu\right),[\omega] \in \partial \mathscr{F}_{n}$, one has

$$
\left(T_{z}(f+g)\right)([\omega])=\left(T_{z}(f)\right)([\omega])+\left(T_{z}(g)\right)([\omega])
$$

by definition. Thus $T_{z}$ is linear. It is also bounded, since

$$
\left\|T_{z}\right\|_{\infty}=\sup _{\|f\|_{2}=1}\left\|T_{z}(f)\right\|_{2} \leq \sup _{\|f\|_{2}=1}\|f\|_{2} \leq 1 .
$$

Hence $T_{z} \in \mathcal{B}\left(L^{2}\left(\partial \mathscr{F}_{n}, \mathbb{C}, \mu\right)\right)$ for all $z \in \mathscr{F}_{n}$. As $\mathcal{B}\left(L^{2}\left(\partial \mathscr{F}_{n}, \mathbb{C}, \mu\right)\right)$ is a $C^{\star}$-algebra, $T_{z}$ has an adjoint operator $T_{z}^{\star}$, which is the bounded operator satisfying

$$
\left\langle T_{z} f, g\right\rangle=\left\langle f, T_{z}^{\star} g\right\rangle,
$$

for all $f, g \in L^{2}\left(\partial \mathscr{F}_{n}, \mathbb{C}, \mu\right)$.

Fact 4.3. The bounded operator $T_{z}^{\star}$, for $z \in \mathscr{F}_{n}$, is given by

$$
\left(T_{z}^{*} f\right)([\omega])= \begin{cases}0 & \text { if }[\omega] \notin z \partial \mathscr{F}_{n} \\ f\left(\left[\omega^{\prime}\right]\right) & \text { if }[\omega]=z\left[\omega^{\prime}\right] .\end{cases}
$$

Proof. Note that $T_{z}^{\star} f \in L^{2}\left(\partial \mathscr{F}_{n}, \mathbb{C}, \mu\right)$, since

$$
\begin{aligned}
\left\|T_{z}^{\star} f\right\|_{2}^{2} & =\int_{\partial \mathscr{F}_{n}}\left|T_{z}^{\star} f\right|^{2} d \mu \\
& =\int_{z \partial \mathscr{F}_{n}}\left|T_{z}^{\star} f\right|^{2} d \mu \\
& \leq \int_{\partial T_{n}}|f|^{2} d \mu .
\end{aligned}
$$


Let $f, g \in L^{2}\left(\partial \mathscr{F}_{n}, \mathbb{C}, \mu\right)$. Then one has

$$
\begin{aligned}
\left\langle f, T_{z}^{\star} g\right\rangle & =\int_{\partial T_{n}} f\left(\overline{T_{z}^{\star} g}\right) d \mu \\
& =\int_{z \partial T_{n}} f\left(\overline{T_{z}^{\star} g}\right) d \mu \\
& =\int_{z \partial T_{n}}\left(T_{z} f\right) \bar{g} d \mu \\
& \leq \int_{\partial T_{n}}\left(T_{z} f\right) \bar{g} d \mu \\
& =\left\langle T_{z} f, g\right\rangle .
\end{aligned}
$$

where equality (4.24) holds by

$$
f\left(\left[z \omega^{\prime}\right]\right) \overline{T_{z}^{\star} g}\left(\left[z \omega^{\prime}\right]\right)=\left(T_{z} f\right)\left(\left[\omega^{\prime}\right]\right) \bar{g}\left(\left[\omega^{\prime}\right]\right) .
$$

Proposition 4.4. The following identities hold for all $x, y \in S \subseteq \mathscr{F}_{n}$

$$
\begin{aligned}
T_{x}^{\star} T_{y} & =\delta_{x y} ; \\
\sum_{i=1}^{n} T_{X_{i}} T_{X_{i}}^{\star} & =1 .
\end{aligned}
$$

In particular, the $C^{\star}$-algebra $C^{\star}\left(\mathscr{F}_{n}, \mu\right) \subseteq \mathcal{B}\left(L^{2}\left(\partial \mathscr{F}_{n}, \mathbb{C}, \mu\right)\right)$ generated by $\mathscr{F}_{n}$ is isomorphic to the Cuntz algebra $\mathcal{O}_{n}$.

Proof. Let $x, y \in S \subseteq \mathscr{F}_{n}$ and let $f \in L^{2}\left(\partial \mathscr{F}_{n}, \mathbb{C}, \mu\right)$. For any $[\omega] \in \partial \mathscr{F}_{n}$ one has

$$
T_{x}^{\star} T_{y}(f)([\omega])=\delta_{x y} f([\omega])
$$

by Fact 4.3. This proves identity (4.28).

Let $[\omega] \in \partial \mathscr{F}_{n}$. Then there exists $x_{j} \in S$ such that $[\omega] \in x_{j} \partial \mathscr{F}_{n}$. Hence one has

$$
T_{x_{i}} T_{x_{i}}^{\star} f([\omega])=\delta_{i j} f([\omega])
$$

for any $f \in L^{2}\left(\partial \mathscr{F}_{n}, \mathbb{C}, \mu\right)$. This yields the identity (4.29).

\subsection{Finitely 1-generated monoids}

Let $\mathscr{M}$ be a finitely 1-generated $\mathbb{N}_{0}$-graded monoid. Then one has a canonical surjective graded homomorphism $\phi_{\mathscr{M}}: \mathscr{F} \rightarrow \mathscr{M}$, where $\mathscr{F}$ is a finitely generated free monoid (cf. (3.4)), which induces a continuous map $\partial \phi: \partial \mathscr{F} \longrightarrow \partial \mathscr{M}$ (cf. Proposition 3.2). In particular,

$$
\mu_{\mathscr{M}}: \operatorname{Bor}(\partial \mathscr{M}) \longrightarrow \mathbb{R}_{0}^{+}
$$

given by $\left.\mu_{\mathscr{M}}(A)=\mu\left(\partial \phi_{\mathscr{M}}\right)^{-1}(A)\right)$ is a Borel probability measure on $\partial \mathscr{M}$.

For $s \in \mathscr{M}$, define the map $\beta_{s}: \partial \mathscr{M} \rightarrow \partial \mathscr{M}$ by

$$
\beta_{s}([f])=[s f], \quad[f] \in \partial \mathscr{M},
$$


where $(s f)(n)=s \cdot f(n)$ for all $n \in \mathbb{N}, f \in \mathscr{D}(\mathbb{N}, \mathscr{M}, \preceq)$. Then, as $\beta_{s}$ is mapping cones to cones, $\beta_{s}$ is continuous. Hence one has a representation

$$
\beta: \mathscr{M} \rightarrow C(\partial \mathscr{M}, \partial \mathscr{M}) .
$$

For $s \in \mathscr{M}$, let $\beta_{\star, s}: L^{2}(\partial \mathscr{M}, \mathbb{C}, \mu) \rightarrow L^{2}(\partial \mathscr{M}, \mathbb{C}, \mu)$ be the map defined by

$$
\beta_{\star, s}(g)([f])=g\left(\beta_{s}([f])\right)=g([s f]), \quad g \in L^{2}(\partial \mathscr{M}, \mu),[f] \in \partial \mathscr{M} .
$$

Then one has

$$
\begin{aligned}
\left\|\beta_{\star, s}(g)\right\|_{2}^{2} & =\int_{\partial \mathscr{M}} \mid g\left(\left.\beta_{s}([f])\right|^{2} d \mu_{\mathscr{M}}\right. \\
& =\int_{\partial \mathscr{M}}|g([s f])|^{2} d \mu_{\mathscr{M}} \\
& =\int_{s \partial \mathscr{M}}|g([f])|^{2} d \mu_{\mathscr{M}} \\
& \leq \int_{\partial \mathscr{M}}|g([f])|^{2} d \mu_{\mathscr{M}} \\
& =\|g\|_{2}^{2},
\end{aligned}
$$

for all $g \in L^{2}(\partial \mathscr{M}, \mathbb{C}, \mu), s \in \mathscr{M}$. Thus,

$$
\left\|\beta_{\star, s}\right\|=\sup _{\|g\|_{2}=1}\left\|\beta_{\star, s}(g)\right\|_{2} \leq 1
$$

for all $s \in \mathscr{M}$, i.e., $\beta_{\star}, s$ is a bounded operator on $L^{2}(\partial \mathscr{M}, \mathbb{C}, \mu)$. By an argument similar to the one used in the proof of Fact 4.2 one can show that it is also linear. In particular, there exists a representation

$$
\beta \star: \mathscr{M} \rightarrow \mathcal{B}\left(L^{2}(\partial \mathscr{M}, \mathbb{C}, \mu)\right) .
$$

\subsection{Right-angled Artin monoids}

Let $\Gamma=(V, E)$ be a finite undirected graph, i.e. $|V|=n<\infty$ and $E \subseteq \mathscr{P}_{2}(V)$, where $\mathscr{P}_{2}(V)$ denotes the set of subsets of cardinality 2 of $V$. The right-angled Artin monoid associated to $\Gamma$ is the monoid $\mathscr{M}^{\Gamma}$ defined by

$$
\left.\mathscr{M}^{\Gamma}=\langle x \in V| x y=y x \text { if }\{x, y\} \in E\right\rangle^{+} .
$$

Clearly, $\mathscr{M}^{\Gamma}$ is $\mathbb{N}_{0}$-graded and finitely 1-generated. By Luis Paris theorem (cf. [6]), $\mathscr{M}^{\Gamma}$ embeds into the rightangled Artin group $G_{\Gamma}$. Thus $\mathscr{M}^{\Gamma}$ has the left-cancellation property as well as the right-cancellation property. The canonical homomorphism $\phi_{\Gamma}: \mathscr{F}\langle V\rangle \longrightarrow \mathscr{M}^{\Gamma}$ is surjetcive and induces a continuous surjective map

$$
\partial \phi_{\Gamma}: \partial \mathscr{F}\langle V\rangle \longrightarrow \partial \mathscr{M}^{\Gamma}
$$

(cf. Proposition 3.2). We denote by $\mu_{\Gamma}$ : Bor $\left(\partial \mathscr{M}^{\Gamma}\right) \longrightarrow \mathbb{R}_{0}^{+}$the Borel probability measure induced by $\partial \phi_{\Gamma}$, i.e., for $A \in \operatorname{Bor}\left(\partial \mathscr{M}^{\Gamma}\right)$ one has

$$
\mu_{\Gamma}(A)=\mu\left(\partial \phi_{\Gamma}^{-1}(A)\right)
$$

where $\mu$ is the measure defined on $\partial \mathscr{F}\langle V\rangle$ by (4.7).

Definition. Let $\Gamma=(V, E)$ be a graph, and let $\Gamma_{1}=\left(V_{1}, E_{1}\right)$ and $\Gamma_{2}=\left(V_{2}, E_{2}\right)$ be subgraphs of $\Gamma$. We say that $\Gamma$ is bipartitly decomposed by $\Gamma_{1}$ and $\Gamma_{2}$, if $V=V_{1} \sqcup V_{2}$ and

$$
E=E_{1} \sqcup E_{2} \sqcup\left\{\left\{v_{1}, v_{2}\right\} \mid v_{1} \in V_{1}, v_{2} \in V_{2}\right\} .
$$

In this case we will write $\Gamma=\Gamma_{1} \vee \Gamma_{2}$. If no such decomposition exists, $\Gamma$ will be called coconnected. 
Any graph $\Gamma$ can be decomposed into connected components $\Gamma_{i}$, i.e. $\Gamma=\sqcup_{i \in I} \Gamma_{i}$. In a similar fashion one may define a decomposition in coconnected components.

Definition. Let $\Gamma=(V, E)$ be a graph and let $\Gamma^{\mathrm{op}}=\bigsqcup_{i \in I} \Lambda_{i}$ be the decomposition of $\Gamma^{\mathrm{op}}$ in its connected components. We will call

$$
\Gamma=\bigvee_{i \in I} \Lambda_{i}^{\mathrm{op}},
$$

the decomposition of $\Gamma$ in coconnected components.

One has the following property.

Fact 4.5. Let $\Gamma=(V, E)$ be an undirected graph. Then $\Gamma$ is coconnected if, and only if, $\Gamma^{\mathrm{op}}$ is connected. In particular, if $\Gamma^{\mathrm{op}}=\bigsqcup_{i \in I} \Lambda_{i}$ is the decomposition of $\Gamma^{\mathrm{op}}$ in its connected components, then one has

$$
\Gamma=\bigvee_{i \in I} \Lambda_{i}^{\mathrm{op}}
$$

where $\Lambda_{i}^{\mathrm{op}}$ are coconnected subgraphs of $\Gamma$.

Proof. Obviously, the graph $\Gamma=\Gamma_{1} \vee \Gamma_{2}$ is bipartitly decomposed if, and only if, $\Gamma^{\mathrm{op}}=\Gamma_{1}^{\mathrm{op}} \sqcup \Gamma_{2}^{\mathrm{op}}$ is not connected. This yields to the claim.

Note that the decomposition in coconnected components implies that ant two vertices in different components must be connected by an edge. From this property one concludes the following straightforward fact.

Fact 4.6. Let $\Gamma=(V, E)$ be a finite graph with unoriented edges, and let $\Gamma=\bigvee_{1 \leq i \leq r} \Gamma_{i}$ be its decomposition in coconnected components, $\Gamma_{i}=\left(V_{i}, E_{i}\right)$.Then

$$
\mathscr{M}^{\Gamma}=\mathscr{M}^{\Gamma_{1}} \times \cdots \times \mathscr{M}^{\Gamma_{r}},
$$

where $\mathscr{M}^{\Gamma_{i}}=\left\langle v \in V_{i}\right\rangle$. In particular, $\partial \mathscr{M}^{\Gamma}=\times_{1 \leq r} \partial \mathscr{M}^{\Gamma_{i}}$ and

$$
L^{2}\left(\partial \mathscr{M}^{\Gamma}, \mathbb{C}, \mu_{\Gamma}\right)=L^{2}\left(\partial \mathscr{M}^{\Gamma_{r}}, \mathbb{C}, \mu_{\Gamma_{1}}\right) \widehat{\otimes} \cdots \widehat{\otimes} L^{2}\left(\partial \mathscr{M}^{\Gamma_{r}}, \mathbb{C}, \mu_{\Gamma_{r}}\right) .
$$

In [2], J. Crisp and M.Laca has shown the following.

Theorem 4.7 ([2], Theorem 6.7). Let $\Gamma=(V, E)$ be a finite unoriented graph such that $\Gamma^{\mathrm{op}}$ has no isolated vertices, and let $\Gamma=\bigvee_{i=1}^{r} \Gamma_{i}$ be the decomposition of $\Gamma$ in coconnected components, $\Gamma_{i}=\left(V_{i}, E_{i}\right)$. Then the universal $C^{\star}$-algebra with generators $\left\{S_{x} \mid x \in V\right\}$ subject to the relations

(i) $S_{x}^{\star} S_{x}=1$ for each $x \in V$;

(ii) $S_{x} S_{y}=S_{y} S_{x}$ and $S_{x}^{*} S_{y}=S_{y} S_{x}^{*}$ if $x$ and $y$ are adjacent in $\Gamma$;

(iii) $S_{x}^{*} S_{y}=0$ if $x$ and $y$ are distinct and not adjacent in $\Gamma$;

(iv) $\prod_{x \in V_{i}}\left(1-S_{x} S_{x}^{\star}\right)=0$ for each $i \in\{1, \ldots, r\}$;

is canonically isomorphic to the boundary quotient $\partial C_{\lambda}\left(\mathscr{M}^{\Gamma}\right)$ for $\mathscr{M}^{\Gamma}$ and it is a simple $C^{\star}$-algebra.

Hence, one has the following proposition.

Proposition 4.8. The $C^{\star}$-algebra $C^{*}\left(\mathscr{M}^{\Gamma}, \mu_{\Gamma}\right)\left(c f\right.$. (1.6)) of a right-angled Artin monoid $\mathscr{M}^{\Gamma}$ is isomorphic to the boundary quotient $\partial C_{\lambda}\left(\mathscr{M}^{\Gamma}\right)$ of Theorem 4.7 .

Proof. Let $\Gamma=(V, E)$ be a finite unoriented graph such that $|V|=n$ and let $\Gamma=\bigvee_{i=1}^{r} \Gamma_{i}$ be its decomposition in coconnected components. It is straightforward to verify (i)-(iii) for the set of operators $\left\{T_{x} \mid x \in V\right\}$, where the operator $T_{X} \in \mathcal{B}\left(L^{2}\left(\partial \mathscr{M}^{\Gamma}, \mathbb{C}, \mu_{\Gamma}\right)\right), \mu_{\Gamma}$ as in (4.45), is defined by

$$
T_{x}(f)([\omega])=f([x \omega]),
$$


and the adjoint operators are given by

$$
\left(T_{\chi}^{\star} f\right)([u])= \begin{cases}0 & \text { if }[u] \notin x \partial \mathscr{M}^{\Gamma} \\ f\left(\left[u^{\prime}\right]\right) & \text { if }[u]=x\left[u^{\prime}\right],\end{cases}
$$

where $f \in L^{2}\left(\partial \mathscr{M}^{\Gamma}, \mathbb{C}, \mu_{\Gamma}\right)$. It remains to prove that it also satisfies (iv). Let

$$
\mathbf{e}_{i}=\prod_{x \in V_{i}}\left(1-T_{X} T_{x}^{\star}\right) .
$$

In order to show that $\mathbf{e}_{i}(f)=0$ for all $f \in L^{2}\left(\partial \mathscr{M}^{\Gamma}, \mathbb{C}, \mu_{\Gamma}\right)$ it suffices to show that $\mathbf{e}_{i}(f)=0$ for $f=f_{1} \otimes \cdots \otimes f_{r}$, $f_{i} \in L^{2}\left(\partial \mathscr{M}^{\Gamma_{i}}, \mathbb{C}, \mu_{\Gamma_{i}}\right)($ cf. (4.50)). Note that

$$
\left(1-T_{\chi} T_{x}^{\star}\right)(f)([u])= \begin{cases}0 & \text { if }[u] \in x \partial \mathscr{M}^{\Gamma}, \\ f([u]) & \text { otherwise. }\end{cases}
$$

Let $[u]=\left[u_{1}\right] \cdots\left[u_{r}\right],\left[u_{j}\right] \in \partial \mathscr{M}^{\Gamma_{j}}$. Then there exists $y \in V_{i}$ such that $\left[u_{i}\right] \in y \partial \mathscr{M}^{\Gamma_{i}}$. Hence, by (4.54)

$$
\left(1-T_{y} T_{y}^{\star}\right)(f)([u])=0 .
$$

Hence $\mathbf{e}_{i}(f)=0$ and this yields the claim.

\section{Fractals}

Let $\mathscr{M}$ be a finitely 1-generated monoid. By an $\mathscr{M}$-fractal we will understand a compact metric space $(X, d)$ with a contracting left $\mathscr{M}$-action $\alpha: \mathscr{M} \longrightarrow C(X, X)$, i.e., there exists a real number $\delta<1$ such that for all $x, y \in X$ and all $\omega \in \mathscr{M} \backslash\{1\}$ one has

$$
d(\alpha(\omega)(x), \alpha(\omega)(y))<\delta \cdot d(x, y) .
$$

The real number $\delta$ will be called the contraction constant. To the authors knowledge the following important question has not been discussed in the literature yet.

Question 4. For which finitely 1-generated monoids $\mathscr{M}$ does there exist an $\mathscr{M}$-fractal $(X, d, \alpha)$ ?

Example 5.1. Let $s_{1}, s_{2}: I \rightarrow I, I=[0,1]$, be defined by $s_{1}(x)=\frac{1}{3} x, s_{2}(x)=\frac{2}{3}+s_{1}(x)$. Then $\left\langle s_{1}, s_{2}\right\rangle \subseteq C(I, I)$ is isomorphic to the free monoid $\mathscr{F}_{2}$ on 2 generators. The $\mathscr{F}_{2}$-fractal $(I, d, \alpha)$, where $d$ is the standard metric and $\alpha$ is the action described above, has as attractor the Cantor set (see [3], Ex. 3.3).

\subsection{The action of the universal boundary on an $\mathscr{M}$-fractal}

Let $\mathscr{M}$ be a finitely 1-generated monoid with grading $\left|\_\right|: \mathscr{M} \rightarrow \mathbb{N}_{0}$. For a strictly decreasing sequence $f \in$ $\mathscr{D}(\mathbb{N}, \mathscr{M}, \preceq)$ and for $n, m \in \mathbb{N}_{0}, m>n$, there exists $\tau_{m, n} \in \mathscr{M} \backslash\{1\}$ such that $f(m)=f(n) \cdot \tau_{m, n}$. By induction, one concludes that $|f(n)| \geq n$. If $[f] \in \partial \mathscr{M}$, then $f$ can be represented by a strictly decreasing sequence (cf. Fact 2.3).

As $\alpha$ is contracting, one concludes that $(\alpha(f(n))(x))$ is a Cauchy sequence for every strictly decreasing sequence $f \in \mathscr{D}(\mathbb{N}, \mathscr{M}, \preceq)$ and thus has a limit point $\alpha(f)(x)=\lim _{n \rightarrow \infty}(\alpha(f(n))(x))$. In more detail, if $\alpha$ has contracting constant $\delta<1$, one has for $n, m \in \mathbb{N}, m>n$, that

$$
d(\alpha(f(m))(x), \alpha(f(n))(x))<\delta^{|f(n)|} \cdot d\left(\alpha\left(\tau_{m, n}\right)(x), x\right) \leq \delta^{|f(n)|} \cdot \operatorname{diam}(X),
$$

where $\operatorname{diam}(X)=\max \{d(y, z) \mid y, z \in X\}$. Thus one has a map

$$
-_{-}: \mathscr{D}(\mathbb{N}, \mathscr{M}, \prec) \times X \longrightarrow X
$$

given by $[f] \cdot x=\alpha(f)(x)$. This map has the following property. 
Remark 5.1. (a) Let $(X, d)$ be a compact metric space. For $A, B \subseteq X$ the Hausdorff metric $\mathfrak{d}: \mathscr{P}(X) \times \mathscr{P}(X) \rightarrow \mathbb{R}_{0}^{+}$, where $\mathscr{P}(X)$ denotes the set of subsets of $X$, is given by

$$
\mathfrak{d}(A, B)=\sup \{d(a, B), d(b, A) \mid a \in A, b \in B\},
$$

where $d(a, B)=\inf \{d(a, b) \mid b \in B\}$ (cf. [3, (2.4)]).

(b) Let $\mathscr{M}$ be a finitely 1-generated monoid, and let $((X, d), \alpha)$ be an $\mathscr{M}$-fractal with attractor $K \subseteq X$. For $\mathscr{S}: \mathscr{P}(X) \rightarrow \mathscr{P}(X)$ given by

$$
\mathscr{S}(A)=\bigcup_{\sigma \in \mathscr{M}_{1}} \alpha(\sigma)(A),
$$

it is well known that $\left(\mathscr{S}^{k}(A)\right)_{k \in \mathbb{N}}$, where $\mathscr{S}^{k}(A)=\mathscr{S}\left(\mathscr{S}^{k-1}(A)\right.$ ), converges to $K$ in the Hausdorff metric (cf. [3, Statement (1)]).

Proposition 5.2. Let $\mathscr{M}$ be a finitely 1-generated monoid, and let $((X, d), \alpha)$ be an $\mathscr{M}$-fractal with attractor $K \subseteq X$. Then the map (5.3) is continuous and $[f] \cdot x \in K$ for all $f \in \mathscr{D}(\mathbb{N}, \mathscr{M}, \prec)$ and $x \in X$.

Proof. Let $f \in \mathscr{D}(\mathbb{N}, \mathscr{M}, \prec)$ be a strictly decreasing function. For $A=\{x\}$, and $\mathscr{S}$ as above, the sequence $\left(\mathscr{S}^{k}(A)\right)_{k \in \mathbb{N}}$ converges to $K$ in the Hausdorff metric. Thus for all $\varepsilon>0$ there exists $N(\varepsilon) \in \mathbb{N}$ such that for all $n>N(\varepsilon)$ one has $\mathfrak{d}\left(\mathscr{S}^{n}(A), K\right)<\varepsilon$. Hence $d(\alpha(f(n))(x), K)<\varepsilon$ for all $n>N(\varepsilon)$, and $\alpha(f)(x)$ is a clusterpoint of $K$. As $K$ is closed this implies $\alpha(f)(x) \in K$.

The map (5.3) is obviously continuous in the second argument. Moreover, let $f, h \in \mathscr{D}(\mathbb{N}, \mathscr{M}, \prec), f, h \prec \tau$, $\tau \in \mathscr{M}$. Then

$$
d(\alpha(f)(x)), \alpha(h)(x)) \leq 2 \cdot \delta^{|\tau|} \cdot \operatorname{diam}(X)
$$

Thus (5.3) is continuous.

Proposition 5.3. Let $f, h \in \mathscr{D}(\mathbb{N}, \mathscr{M}, \prec)$ satisfying $f \preceq h$. Then, $\alpha(f)(x)=\alpha(h)(x)$.

Proof. We may assume that $f(n) \preceq h(n)$ for all $n \in \mathbb{N}$, i.e., there exists $y_{n} \in \mathscr{M}$ such that $f(n)=h(n) \cdot y_{n}$. Then, by the same argument which was used for (5.2), one concludes that

$$
d\left(\alpha(f(n))(x), \alpha(h(n)(x)) \leq \delta^{|h(n)|} \operatorname{diam}(X) \leq \delta^{n} \operatorname{diam}(X) .\right.
$$

This yields the claim.

From Proposition 5.3 one concludes that the map (5.3) induces a map

$$
\text { - } \_: \widetilde{\partial} \mathscr{M} \times X \longrightarrow X
$$

given by $\pi([f]) \cdot x=\alpha(f)(x)$ (cf. (2.13)), and thus an action of $\widetilde{\partial} \mathscr{M}$ on $X$.

The following property suggest to think of $\left(\widetilde{\partial} \mathscr{M}, \mathscr{T}_{c}\right)$ as the universal attractor of an $\mathscr{M}$-fractal.

Proposition 5.4. Let $x \in X$, and let $K \subset X$ be the attractor of the $\mathscr{M}$-fractal $((X, d), \alpha)$. Then the induced map

$$
\kappa_{x}: \partial \mathscr{M} \longrightarrow K
$$

given by $\kappa_{x}([f])=\alpha(f)(x)$ is surjective.

Proof. Let $z \in K$, and $A=\{x\}$. By (cf. [3, (2.4)]), for all $\varepsilon>0$ there exists $N(\varepsilon) \in \mathbb{N}$ such that for all $n>N(\varepsilon$ ) one has $\mathfrak{d}\left(\mathscr{S}^{n}(A), z\right)<\varepsilon$, i.e., there exists a sequence $\left(f_{n}\right)_{n \in \mathbb{N}}, f_{n} \in \mathscr{M}_{n}, f_{n+1} \in \bigcup_{\sigma \in \mathscr{M}_{1}}\left\{\sigma \cdot f_{n}\right\}$, such that $d\left(\alpha\left(f_{n}\right)(x), z\right)<\varepsilon$.

If $\mathscr{M}$ is $\mathscr{T}$-regular, then $\left(\overline{\mathscr{M}}, \mathscr{T}_{c}(\overline{\mathscr{M}})\right)$ is compact (cf. Proposition 4.1). Hence $\left(f_{n}\right)_{n \in \mathbb{N}}$ has a cluster point $f \in \overline{\mathscr{M}}$. As $\left|f_{n}\right|=n$, one has $f \notin \mathscr{M}$ and thus $f \in \partial \mathscr{M}$. It is straightforward to verify that $[f] \cdot x=z$, showing that $\kappa_{X}$ is surjective. 


\subsection{The $C^{\star}$-algebra associated to an $\mathscr{M}$-fractals for a finitely 1 -generated monoid}

Let $\mathscr{M}$ be a finitely 1-generated monoid, and let $((X, d), \alpha)$ be an $\mathscr{M}$-fractal with attractor $K$. For $x \in X$ there exists a continuous mapping $\kappa_{X}: \partial \mathscr{M} \rightarrow K$ (cf. Theorem C). Let $\mu_{X}$ : Bor $(K) \rightarrow \mathbb{R}_{0}^{+}$be the probability measure given by (1.8). Then $\mathscr{M}$ acts on $K$, and thus also on $L^{2}\left(K, \mathbb{C}, \mu_{x}\right)$.

For $t \in \mathscr{M}$ let $\gamma_{t}: L^{2}\left(K, \mathbb{C}, \mu_{x}\right) \rightarrow L^{2}\left(K, \mathbb{C}, \mu_{x}\right)$ be given by

$$
\gamma_{t}(g)(x)=g\left(\alpha_{t}(x)\right)
$$

where $g \in L^{2}\left(K, \mathbb{C}, \mu_{x}\right)$. Hence the monoid $\mathscr{M}$ acts on the Hilbert space $L^{2}\left(K, \mathbb{C}, \mu_{x}\right)$ by bounded linear operators.

$$
\left\|\gamma_{t}(g)\right\|_{2}^{2}=\int_{K}\left|\gamma_{t}(g(z))\right|^{2} d \mu_{x}=\int_{K}\left|g\left(\alpha_{t}(z)\right)\right|^{2} d \mu_{x} \leq\|g\|_{2}^{2}
$$

(cf. $§ 4.1)$. One defines the $C^{\star}$-algebra generated by the $\mathscr{M}$-fractal $((X, d), \alpha)$ by

$$
C^{\star}\left(\mathscr{M}, X, d, \mu_{x}\right)=\left\langle\gamma_{t}, \gamma_{t}^{\star} \mid t \in \mathscr{M}\right\rangle \subseteq \mathcal{B}\left(L^{2}\left(K, \mathbb{C}, \mu_{x}\right)\right) .
$$

\section{References}

[1] N. Bourbaki, General topology. Chapters 1-4, Elements of Mathematics (Berlin), Springer-Verlag, Berlin, 1998, Translated from the French, Reprint of the 1989 English translation. MR 1726779

[2] J. Crisp and M. Laca, Boundary quotients and ideals of Toeplitz $C^{\star}$-algebras of Artin groups, J. Funct. Anal. 242 (2007), no. 1, 127-156. MR 2274018

[3] J. E. Hutchinson, Fractals and self-similarity, Indiana Univ. Math. J. 30 (1981), no. 5, 713-747. MR 625600

[4] X. Li, Semigroup $C^{\star}$-algebras, arXiv:1707.05940v1 (2017).

[5] X. Li, T. Omland, and J. Spielberg, $C^{\star}$-algebras of right LCM one-relator monoids and Artin-Tits monoids of finite type, preprint, July 2018.

[6] L. Paris, Artin monoids inject in their groups, Comment. Math. Helv. 77 (2002), no. 3, 609-637. MR 1933791 